\title{
Ruxolitinib in Glucocorticoid Refractory Chronic Graft versus Host Disease
}

\author{
Aparna Sharma ${ }^{1}$ Vibhor Sharma $^{2}$ \\ ${ }^{1}$ Department of Medical Oncology, Dr. B.R.A. IRCH, All India Institute \\ of Medical Sciences, New Delhi, India \\ 2 Medical Oncology, Hematology and Bone Marrow Transplant, Paras \\ Hospitals, Gurugram, Haryana, India
}

Ind J Med Paediatr Oncol 2021;42:582-583.

The phase 3 REACH 3 trial $^{1}$ investigates ruxolitinib compared with investigator's choice of therapy for glucocorticoid refractory or dependent moderate to severe chronic graft versus host disease (GVHD). This REACH 3 study was done in patients who were 12 years or older with moderate to severe glucocorticoid refractory or dependent chronic GVHD. The drugs administered in investigator's choice of therapy arm include everolimus, extracorporeal phototherapy, ibrutinib, imatinib, infliximab, low-dose methotrexate, mycophenolate mofetil, sirolimus, and rituximab. REACH 3 study shows improvement in overall response at 24 weeks in the patients' arm administered with ruxolitinib compared with the control cohort $(49.7 \%$ vs $25.6 \%$, odds ratio 2.99 , $p<0.001$ ). Ruxolitinib also led to greater median failure-free survival ( $>18.6$ vs. 5.7 months, hazard ratio $0.37, p<0.001$ ) and higher symptom response ( $24.2 \%$ vs $11 \%$, odds ratio 2.62 , $p=0.001$ ). The dose of ruxolitinib used was $10 \mathrm{mg}$ twice daily (bd). In REACH $1,^{2}$ a phase 2 study investigating the role of ruxolitinib in patients 12 years or older with grades 2 to 4 steroid refractory acute GVHD, the starting dose of ruxolitinib was $5 \mathrm{mg}$ bd for first 3 days. It also included an option to increase the dosage to $10 \mathrm{mg}$ bd in absence of cytopenia. In REACH 2 study, $^{3}$ a phase 3 study evaluating ruxolitinib in patients 12 years or older with grades 2 to 4 steroid refractory acute GVHD, the planned dose of ruxolitinib was $10 \mathrm{mg}$ bd. The median dose administered was $16.8 \mathrm{mg}$ in two divided doses. In REACH 1 study, ruxolitinib could be tapered after day 180 in patients who achieved complete response or very good partial response in acute GVHD and had discontinued corticosteroids for at least 8 weeks. In REACH 2 study, tapering of ruxolitinib was allowed after day 56 in the responsive patients. In REACH 1 study, corticosteroids
Address for correspondence Vibhor Sharma, MD, DM, Senior Consultant, Medical Oncology, Hematology and Bone Marrow Transplant, Paras Hospitals, Gurugram 122002, Haryana, India (e-mail: drvibhoroncology@gmail.com).

were tapered as per institutional guidelines, with physician discretion allowed. In REACH 2 study, continuation of glucocorticoids was allowed alongside ruxolitinib or control therapy. Wu et $\mathrm{al}^{4}$ have reported a weight-based dosing of ruxolitinib in chronic GVHD patients wherein patients having a weight more than $60 \mathrm{~kg}$ received $10 \mathrm{mg}$ bd while patients $\leq 60 \mathrm{~kg}$ received $5 \mathrm{mg}$ bd.

In REACH 3 study, $16.4 \%$ patients discontinued ruxolitinib due to adverse events. In REACH 3 study, grade $\geq 3$ thrombocytopenia and anemia was noted in $15.2 \%$ and $12.7 \%$ of patients respectively in ruxolitinib arm and $10.1 \%$ and $7.6 \%$ of patients respectively in the arm receiving control therapy. The incidence of cytomegalovirus infections was $5.5 \%$ and $8.2 \%$ in ruxolitinib and control arm respectively. The incidence of grade $\geq 3$ pneumonia in the patients of ruxolitinib arm and control arm was $8.5 \%$ and $9.5 \%$ respectively.

The authors in REACH 3 study have not reported the median dose of ruxolitinib administered. It is recommended that dose of ruxolitinib be reduced when coadministered with fluconazole. ${ }^{5,6}$ It would be worthwhile to have the author's opinions regarding the use of lower-dose ruxolitinib coadministered with fluconazole, especially in resource constrained settings. The primary end point of REACH 3 study was the overall response at week 24 . No reasoning has been given as to why week 24 was chosen as the end time point. In REACH 3 study, a higher overall response was seen in ruxolitinib arm than in control arm regardless of the organs involved. However, the response rates in lung and liver were unsatisfactory in both the patient arms. The response rate in lungs was 8.6 and $6.1 \%$ respectively in ruxolitinib and control arms. The response rate in liver was 24.4 and $21.7 \%$ respectively in ruxolitinib and control arm. This demonstrates that
DOI https://doi.org/ 10.1055/s-0041-1740950. ISSN 0971-5851.
(C) 2021. Indian Society of Medical and Paediatric Oncology. All rights reserved.

This is an open access article published by Thieme under the terms of the Creative Commons Attribution-NonDerivative-NonCommercial-License, permitting copying and reproduction so long as the original work is given appropriate credit. Contents may not be used for commercial purposes, or adapted, remixed, transformed or built upon. (https://creativecommons.org/ licenses/by-nc-nd/4.0/)

Thieme Medical and Scientific Publishers Pvt. Ltd., A-12, 2nd Floor, Sector 2, Noida-201301 UP, India 
treatment of chronic GVHD in case of the recovery of these organs can be very difficult. Over $50 \%$ of patients enrolled in this trial had acute GVHD. The authors have included patients who were treated with ruxolitinib for steroid refractory acute GVHD. The number of such patients and the response rates of ruxolitinib in this subset in chronic GVHD would be interesting to know. In REACH 3, patients treated previously with two or more systemic therapies for chronic GVHD in addition to glucocorticoids with or without calcineurin inhibitors were ineligible. Wu et $\mathrm{al}^{4}$ included patients who had received 1-6 lines of second-line therapies for chronic GVHD. The median time taken to respond to ruxolitinib and the data regarding nonresponders or patients who had early deteriorating effects from ruxolitinib arm in REACH 3 study would further aid in our understanding. The overall survival in the ruxolitinib responders versus nonresponders would also be interesting. The limitations of REACH 3 study include the absence of a strong end point such as glucocorticoid-free remission, and the presence of confounders including concomitant treatments. This makes the determination of the effect on glucocorticoid dose over time with ruxolitinib as compared with commonly used therapies difficult.

REACH 3 study leads to important practice-changing conclusions, especially regarding the use of ruxolitinib in steroid refractory chronic GVHD. In resource-limited settings as in our country, the administration of ruxolitinib with azole antifungals may help in reducing the dose and hence the financial burden. The use of ruxolitinib should be started earlier rather than later in the case of steroid refractory chronic GVHD.

Funding

None.

Conflict of Interest

None.

\section{References}

1 Zeiser R, Polverelli N, Ram R, et al; REACH3 Investigators. Ruxolitinib for glucocorticoid-refractory chronic graft-versus-host disease. N Engl J Med 2021;385(03):228-238

2 Jagasia M, Perales MA, Schroeder MA, et al. Ruxolitinib for the treatment of steroid-refractory acute GVHD (REACH1): a multicenter, open-label phase 2 trial. Blood 2020;135(20): 1739-1749

3 Zeiser R, von Bubnoff N, Butler J, et al; REACH2 Trial Group. Ruxolitinib for glucocorticoid-refractory acute graft-versus-host disease. N Engl J Med 2020;382(19):1800-1810

$4 \mathrm{Wu} \mathrm{H}$, Shi J, Luo Y, et al. Evaluation of ruxolitinib for steroidrefractory chronic graft-vs-host disease after allogeneic hematopoietic stem cell transplantation. JAMA Netw Open 2021;4(01): e2034750

5 Aslanis V, Umehara K, Huth F, et al. Multiple administrations of fluconazole increase plasma exposure to ruxolitinib in healthy adult subjects. Cancer Chemother Pharmacol 2019;84(04): 749-757

6 Food and Drug Administration. Jakafi (ruxolitinib) tablets. Reference ID: 4191667. https://www.accessdata.fda.gov/drugsatfda_docs/label/2017/202192s015lbl.pdf. Accessed December 8, 2021 